\section{SAT0656 OPTICAL IMAGING OF PHAGOCYTE MIGRATION REPRESENTS A NOVEL METHOD TO DETERMINE DISEASE ACTIVITY IN CIA}

S. Gran ${ }^{1}$, L. Honold ${ }^{2}$, O. Fehler ${ }^{1}$, S. Zenker ${ }^{1}$, S. Hermann ${ }^{2}$, M. Schäfers ${ }^{2}$, T. Vogl ${ }^{1}$, J. Roth ${ }^{1} .{ }^{1}$ Institute of Immunology; ${ }^{2}$ European Institute for Molecular Imaging, Münster, Germany

Background: Recruitment and migration of phagocytes to the site of inflammation are key events in the onset of inflammation. Albeit crucial for pathogen elimination, tissue repair and restoration of tissue homeostasis, dysregulated phagocyte infiltration can also cause severe inflammatory disorders. Therefore, targeting and modulation of phagocyte infiltration represents a promising new approach to fight inflammatory disorders and diseases, such as rheumatoid arthritis. Additionally, non-invasive tracking of phagocyte migration to the site of inflammation could extend both scientific knowledge as well as the repertoire of diagnostic strategies in clinical use.

Objectives: The aim of this study was to establish a fluorescence reflectance imaging (FRI) based system to visualize and analyze migration properties of different cell populations in inflammatory disease models, like experimental arthritis, in vivo.

Methods: Immortalized murine myeloid progenitor ER-HoxB8 cells were differentiated to neutrophils or monocytes (1). Cells were labeled with the membraneselective fluorescent dyes DIR (2) or DID, respectively. We analyzed viability and functionality of stained cells in vitro and investigated their ability to migrate to sites of inflammation in vivo in several mouse models - particularly in a collagen induced arthritis (CIA) mouse model - via fluorescence reflectance imaging (FRI). Using CRISPR-Cas9 technology we introduced targeted gene deletions for main adhesion molecules.

Results: Differentiated ER-HoxB8 cells could effectively be labeled with DIR or DID. Labeling of monocytes or neutrophils did not affect cellular viability or functionality in vitro. Subsequent in vivo imaging experiments allowed the visualization of migrated labeled phagocytes in different murine disease models, thereby cells could be detected at sites of inflammation with high sensitivity and specificity. In a CIA mouse model the amount of immigrated cells could even be associated closely to disease score and disease severity. Thus, the detection of immigration of labeled cells might also give hints about new inflammatory spots that are about to settle up before they can be detected macroscopically. Furthermore, differential cell labeling allowed direct quantitative comparison of differences in migration rates of wildtype and CD18 or CD49d knockout cells in vivo.

Conclusions: Specific and distinguishable labeling of diverse cell types allows in vivo tracking and subsequent quantification of migrated cells within the same animal. Targeted gene deletion allows analysis of molecular mechanisms relevant for leukocyte recruitment during different stages of arthritis. Correlation of the amount of immigrated cells to disease severity offers new opportunities to non-invasively detect and monitor inflammatory sites in vivo.

\section{References:}

[1] Wang GG, Calvo KR, Pasillas MP, Sykes DB, Häcker H, Kamps MP. Quantitative production of macrophages or neutrophils ex vivo using conditional Hoxb8. Nat Methods. 2006;3(4):287-93.

[2] Eisenblätter M, Ehrchen J, Varga G, Sunderkötter C, Heindel W, Roth J, et al. In vivo optical imaging of cellular inflammatory response in granuloma formation using fluorescence-labeled macrophages. J Nucl Med. 2009;50:1676-82.

Disclosure of Interest: None declared

DOI: 10.1136/annrheumdis-2017-eular.6151

\section{SAT0657 CAN VASCULAR INFLAMMATION IN RA BE DETECTED USING} CONTRAST ENHANCED MRI? PRELIMINARY RESULTS

S. Skeoch ${ }^{1,2}$, R. Little ${ }^{3}$, Y. Watson ${ }^{4}$, S. Cheung ${ }^{3}$, J. O'Connor ${ }^{3}$, Y. Alexander ${ }^{5}$, I. Bruce ${ }^{1,2}$, G. Parker ${ }^{3}$, J. Waterton ${ }^{3} .{ }^{1}$ Manchester NIHR Musculoskeletal Biomedical Research Unit, Central Manchester University Hospitals NHS Foundation Trust; ${ }^{2}$ Centre for Epidemiology; ${ }^{3}$ Centre for Imaging Sciences; ${ }^{4}$ University of Manchester; ${ }^{5}$ Health Related Research, Manchester Metropolitan University, Manchester, United Kingdom

Background: In rheumatoid arthritis (RA) vascular inflammation may contribute to excess cardiovascular (CV) risk. Contrast enhanced MRI has been used to detect vascular inflammation in Takayasu's arteritis. In RA, its utility has not been studied but it may provide a method of identifying and monitoring vascular inflammation and effects of therapy.

Objectives: We sought to compare carotid artery wall enhancement on contrast enhanced MRI in RA patients and controls and evaluate association with circulating markers of inflammation, endothelial activation and $\mathrm{CV}$ risk factors. Methods: Patients and age/sex matched controls underwent clinical and serological assessment (details in Table 1) and a screening carotid ultrasound. Those with wall thickening $>2 \mathrm{~mm}$ had a carotid MRI. $\mathrm{T}_{1}$-weighted images were acquired before and after gadopentate injection on a 3T scanner. Increase in mean signal intensity (SI) in the carotid vessel wall normalised to adjacent skeletal muscle provided a global enhancement metric $\left(\mathrm{WE}_{\mathrm{G}}\right)$. Histograms of distribution of Sls on pre-and post- contrast images were generated and bimodal Gaussian distributions fitted to explore patterns of enhancement within the wall. MRI measurements were compared between groups and association with serological markers tested using non-parametric statistics.
Results: 27 patients and 10 controls underwent MR imaging. Key characteristics are seen in Table 1. There was no difference in $\mathrm{WE}_{\mathrm{G}}$ between groups and no association with serological markers. However a bimodal distribution of SI in vessel wall was observed (Figure 1) and in exploratory analysis these two components were analysed separately. For the low-signal component enhancement (WE $\mathrm{E}_{\text {low }}$ ) correlated with ESR, I-CAM, e-selectin $(r=0.39, p=0.03 ; r=0.44, p=0.01 ; r=0.37, p=0.04)$. There was a trend towards correlation with CRP $(r=0.30,0.08)$ and towards higher values in patients (median $0.20(0.15,0.33)$ vs $0.14(0.11,0.22), \mathrm{p}=0.051)$. Enhancement in the high-signal component $\left(\mathrm{WE}_{\text {high }}\right)$ was not associated with serological markers or RA status.

Table 1.Key characteristics (median (IQR) or frequency (\%) where*)

\begin{tabular}{lccc}
\hline & Cases & Controls & P value \\
\hline Age $(\mathrm{yrs})$ & $61.0(56.5,64.6)$ & $55.4(54.0,57.0)$ & 0.07 \\
Female & $22(81)$ & $5(50)$ & 0.06 \\
LDL $(\mathrm{mmol} / \mathrm{L})$ & $2.7(2.25,3.23)$ & $3.80(3.24,4.53)$ & 0.08 \\
Glucose $(\mathrm{mmol} / \mathrm{L})$ & $4.8(4.6,5.0)$ & $5.8(5.3,6.0)$ & 0.01 \\
Hs-CRP $(\mathrm{mg} / \mathrm{l})$ & $3.34(2.04,5.75)$ & $0.81(0.39,4.58)$ & 0.047 \\
ESR $(\mathrm{mm} / \mathrm{hr})$ & $18(10,35)$ & $7(5,10)$ & 0.01 \\
ICAM $(\mathrm{ng} / \mathrm{ml})$ & $161.5(134.8,213.4)$ & $151.3(106.9,220.8)$ & 0.51 \\
V-CAM1 $(\mathrm{ng} / \mathrm{ml})$ & $398(352,473)$ & $376(353,390)$ & 0.52 \\
E-selectin $(\mathrm{ng} / \mathrm{ml})$ & $7.55(5.79,21.1)$ & $4.71(3.01,7.76)$ & 0.11 \\
WE $_{G}$ & $0.23(0.23,0.28)$ & $0.25(0.18,0.27)$ & 0.62 \\
WE $_{\text {low }}$ & $0.20(0.15,0.33)$ & $0.14(0.11,0.22)$ & 0.05 \\
WE $_{\text {high }}$ & $0.32(0.22,0.50)$ & $0.33(0.29,0.38)$ & 0.73 \\
\hline
\end{tabular}
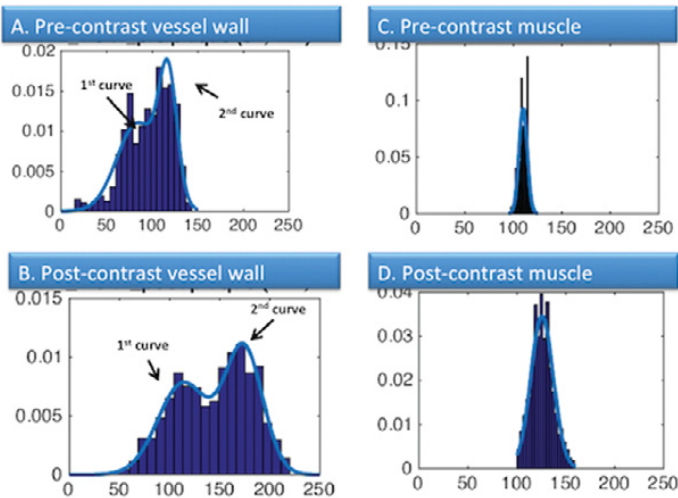

Eigure 1. Distribution of signal intensity (SI) within the vessel wall and muscle referent before and after contrast injection ( $x$ axis signal intensity (a.u), y axis relative frequency). Gaussian curves were fitted and mean of fit values for each curve were calculated. A bimodal distribution with low and high signal intensity components can be seen in ves wall (panel A \& B) compared with a single curve in muscle (panel C\& D). For each curve the difference between pre and post-contrast mist

Conclusions: Simple global SI measurement on MRI did not suggest vascular inflammation in RA. However, the bimodal SI distribution may represent different wall components e.g. intima and adventitia. The differential enhancement, and association of $W_{E_{\text {low }}}$ with inflammation, endothelial activation and $R A$ status, warrants further investigation.

Disclosure of Interest: None declared

DOI: 10.1136/annrheumdis-2017-eular.6657

\section{SAT0658 THE STIFFNESS OF MEDIAN NERVE MEASURED BY ELASTOSONOGRAPHY IN PATIENTS WITH RHEUMATOID ARTHRITIS}

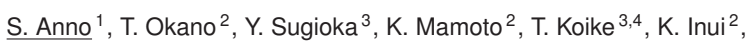
H. Nakamura ${ }^{2} .{ }^{1}$ Yodogawa Christian Hospital; ${ }^{2}$ Department of Orthopedic Surgery, Osaka City University Graduate School of Medicine; ${ }^{3}$ Center for Senile Degenerative Disorders (CSDD), Osaka City University Medical School, Osaka; ${ }^{4}$ Search Institute for Bone and Arthritis Disease (SINBAD), Wakayama, Japan

Background: Carpal tunnel syndrome (CTS) is the most frequent neuropathy of all entrapment neuropathies in the general population. Rheumatoid arthritis (RA) is one of the disease generate secondary CTS. The pathophysiology of CTS of RA might be different from idiopathic CTS. RA is a disease that has the characteristics to generate inflammatory synovial proliferation of the joint and also tendon. Although inflammation of the wrist joint and synovial tissue of the flexor tendons can cause increased pressure in the carpal tunnel, there is a possibility that even RA patients without symptoms of CTS also have subclinical median nerve damage because of the synovial proliferation and inflammation.

Objectives: The aim of this study was to assess and compare the stiffness of the median nerve measured by quantitative elastosonography between patients with RA without symptom of CTS and controls.

Methods: Four hundred two hands in 201 patients with RA and 222 hands in controls were included. All participants were examined both wrists. Ultrasound (US) examination was performed by using a 5- to $18-\mathrm{MHz}$ linear array transducer $(\mathrm{H}$ VISION Ascendus; HitachiAloka Medical,Tokyo, Japan). As a reference medium, an acoustic coupler (EZUTECPL1; HitachiAloka Medical) with a standardized 